\title{
Genetic Divergence, Character Correlations and Heritability Study in 22 Accessions of Groundnut (Arachis hypogaea L.)
}

\author{
Sunday Clement Olubunmi Makinde ${ }^{1} \&$ Omolayo Johnson Ariyo ${ }^{2}$ \\ ${ }^{1}$ Department of Botany, Faculty of Science. Lagos State University, Lagos, Nigeria \\ ${ }^{2}$ College of Plant Science, University of Agriculture Abeokuta, Abeokuta, Nigeria \\ Correspondence: Sunday Clement Olubunmi Makinde, Department of Botany, Faculty of Science, Lagos State \\ University, Lagos. Nigeria. Tel: 234-803-327-7358. E-mail: scmakinde@yahoo.com; \\ olubunmi.makinde@lasu.edu.ng
}

Received: June 25, 2012 Accepted: August 23, 2012 Online Published: September 15, 2012

doi:10.5539/.jps.v2n1p7 URL: http://dx.doi.org/10.5539/jps.v2n1p7

\begin{abstract}
Twenty two groundnut genotypes collected from International Crops Research Institute for Semi Arid Tropics (ICRISAT) and local sources (Samaru, UNILORIN and UNAAB) were cultivated in Lagos $\left(60^{\circ} 36^{\prime} \mathrm{N}, 30^{\circ} 34^{\prime} \mathrm{E}\right)$ and Abeokuta $\left(70^{\circ} 10^{\prime} \mathrm{N}, 30^{\circ} 20^{\prime} \mathrm{E}\right)$ during 2010 planting seasons, to determine the genetic variability and performance of the genotypes. Data collected on ten (10) characters and yield was subjected to multivariate analysis to determine genetic divergence among the genotypes. Number of pods per plant showed significant positive correlation with yield per plant in both environments also had the largest direct positive effect on yield per plant $(0.66,0.70)$. Days to maturity showed the largest direct negative effect of -0.33 and -0.36 in Lagos 2003 and Abeokuta 2004 respectively. Heritability estimates ranged from $62.34 \%$ to 90.67 and 24.75 to 89.46 for number of branches per plant at flowering and days to maturity in (E1) Lagos and (E2) Abeokuta, respectively. The mean squares of the combined analysis of variance revealed significant genotypes and Genotype $\mathrm{x}$ Environment interactions on yield per plant.
\end{abstract}

Keywords: Arachis hypogaea, genetic variability, heritability, character correlation

\section{Introduction}

Successful establishment of germplasm collections and plant introduction for crop improvement as well as for germplasm conservation require studies in genetic variability within plant populations. Jain and Workman (1966) stated that such genetic variability and heterozygosity within populations existed in both natural and agricultural populations. Wright and Dobzhansky (1970) emphasized that the maintenance of this variability depended on complex interactions among a number of genetic and environmental factors. Ariyo (1987a) buttressed this fact further by stating that progress in breeding for economic characters often depends on the availability of a large germplasm representing a diverse genetic variation. He added that for a long term improvement programme, a large and diverse germplasm collection is an invaluable source of parental strains for hybridization and subsequent development of improved varieties. According to White and Gonzalez (1990) accurate cultivar evaluations and ability to differentiate between cultivars in respect of genetic parameters associated with adaptedness in cultivated plants and their wild progenitors are critical to any plant breeding programme.

Food legumes are important sources of protein. For many people, particularly those in developing countries who cannot afford animal products or who do not eat meat for other reasons, pulses form an essential part of their daily diet. The value of legumes not only derives from the amount of protein they contain but also from the fact that legume seed protein complements the deficiency of cereal grain protein (Swaminatham et al., 1969). Negative association between seed yield and seed size has been reported by White and Gonzalez (1990). Therefore, successful selection for improved performance is dependent on obtaining estimates of their genetic value from past evaluations that are predictive of future performance (Clay \& Dombek, 1995). Lack of sufficient information has hindered genetic performance evaluations of crop plant breeding.

The existence of genetic variation can be employed as the basis for improving yield and other potentials of crop plants. Evidence now abounds to show that a great deal of genetic variation and diverse genetic bases necessary for creating further genetic variability exist in groundnut. Improvement of groundnut cultivar is difficult since 
the crop is 100 percent inbred (Smith, 1950; Purseglove, 1975). However, there is a possibility of cross pollination which could range from 0 to $39 \%$, depending on environment, location and genotype (Gibbons \& Tatterfield, 1969). For instance Culp et al. (1968) observed 0.01-0.55 percent outcrossing in Virginia cultivars in USA. Breeders have focused on exploiting local and exotic germplasm by selection rather than hybridization. For example, the cultivars 'Samaru 38' and 'Samaru 68' recommended in Northern Nigeria were improved through pedigree selection from local materials (Kowal \& Kassan, 1978). Therefore, the aim of this study was to determine the extent of genetic divergence and performance in groundnut genotypes

\section{Materials and Methods}

The twenty two genotypes of groundnut used in this study composed of 15 accessions collected from International Crop Research Institute for the Semi-Arid Tropics (ICRISAT), Patancheru, India. The remaining 7 genotypes were collected from different research centers within Nigeria, Table 1 presents the genotype coding with their collection centre. The genotypes were cultivated during the planting season of 2010 in two major locations, viz; Department of Botany, Botanical Garden, Lagos State University-Ojo Campus, Lagos $\left(6^{\circ} 36^{\prime} \mathrm{N}, 3^{\circ}\right.$ 34'E) Lagos State, Nigeria and University of Agriculture, Abeokuta Research and Experimental farm, UNAAB permanent site, Alabata, Abeokuta $\left(7^{\circ} 10^{\prime} \mathrm{N}, 3^{\circ} 20^{\prime} \mathrm{E}\right)$ Ogun State, Nigeria. Each planting is taking to represent an environment.

Table 1. Code names and source/origin of groundnut genotypes used in this study

\begin{tabular}{|c|c|c|}
\hline Number & Genotype & source/origin \\
\hline 1 & $\mathrm{ICG}-4998$ & ICRISAT India \\
\hline 2 & ICG -862 & ICRISAT India \\
\hline 3 & $\mathrm{ICG}-6402$ & ICRISAT India \\
\hline 4 & $\mathrm{ICG}-8490$ & ICRISAT India \\
\hline 5 & $\mathrm{ICG}-4412$ & ICRISAT India \\
\hline 6 & ICG - 156 & ICRISAT India \\
\hline 7 & ICG - 14466 & ICRISAT India \\
\hline 8 & $\mathrm{ICG}-12370$ & ICRISAT India \\
\hline 9 & $\mathrm{ICG}-2106$ & ICRISAT India \\
\hline 10 & $\mathrm{ICG}-4343$ & ICRISAT India \\
\hline 11 & ICG - 12189 & ICRISAT India \\
\hline 12 & $\mathrm{ICG}-442$ & ICRISAT India \\
\hline 13 & $\mathrm{ICG}-4598$ & ICRISAT India \\
\hline 14 & $\mathrm{ICG}-7000$ & ICRISAT India \\
\hline 15 & ICG - 1399 & ICRISAT India \\
\hline 16 & ICGY-6M- 5236 & Zaria, Nigeria \\
\hline 17 & ICG-IS- 11687 & Zaria, Nigeria \\
\hline 18 & ICGY-5M- 4746 & Zaria, Nigeria \\
\hline 19 & ICG-IS- 6646 & UNILORIN, Nigeria \\
\hline 20 & ICG- IS- 3584 & UNILORIN, Nigeria \\
\hline 21 & ICG49- 85A & UNAAB, Nigeria \\
\hline 22 & UGA-7- M & UNAAB, Nigeria \\
\hline
\end{tabular}

Following land preparation, the twenty two genotypes were grown in double-row plots, replicated 3 times in a randomized complete block design. Each row contained ten stands spaced 40 cm apart. An inter-row spacing of $1 \mathrm{~m}$ was maintained ( $1 \mathrm{~m} \times 4 \mathrm{~m}$ plot size). Each stand was thinned to one plant at two weeks after emergence. Manual weeding was done at regular intervals to ensure minimal crop -weeds competition. There was no 
application of inorganic fertilizers and chemicals (herbicides and pesticides) throughout the plantings. Agronomic and yield data were collected on each genotype. Ten competitive plants were sampled in each plot. At maturity, pods were harvested on plant basis and data were collected on yield and ten agronomic characters. Table 2 contains the characters scored for and their method of scoring.

Table 2. Groundnut characters used in this study and their methods of measurement/scoring

\begin{tabular}{lll}
\hline $\mathrm{S} / \mathrm{N}$ & Character & Method of scoring \\
\hline 1 & Days to 50\% flowering & Estimated using calendar \\
2 & Height/ plant at flowering $(\mathrm{cm})$ & Measured \\
3 & Number of leaves/ plant at flowering & Counted \\
4 & Days to maturity & Estimated using calendar \\
5 & Final height/ plant $(\mathrm{cm})$ & Measured \\
6 & Number of branches/ plant at maturity & Counted \\
7 & Nodes on the main stem/ plant at maturity & Counted \\
8 & Stem girth/ plant at maturity $(\mathrm{cm})$ & Measured \\
9 & Number of pods/ plant & Counted \\
10 & Sample seed (100 seeds) weight $(\mathrm{g})$ & Measured \\
11 & Yield/ plant (g) & Measured \\
\hline
\end{tabular}

Data obtained were subjected to analysis of variance following the method of Gomez and Gomez (1984). Variance and covariance components were used to obtain the phenotypic, genotypic and environmental correlations as described below, following the method of Falconer (1989) and Singh (1991).

Phenotypic correlation,

$$
r_{p}=\left(\sigma_{g} x y+\sigma_{e} x y\right) /\left[\left(\sigma_{g}^{2} x+\sigma_{e}^{2} x\right)\left(\sigma_{g}^{2} y+\sigma_{e}^{2} y\right)\right]^{1 / 2}
$$

Genotypic correlation,

$$
r_{g}=\sigma g x y /\left(\sigma_{g}^{2} x \quad x \sigma_{g}^{2} y\right)^{1 / 2}
$$

Environmental correlation,

$$
r_{e}=\sigma e x y /\left(\sigma_{e}^{2} x \quad x \sigma_{e}^{2} y\right)^{1 / 2}
$$

Heritability estimate,

$$
\mathrm{h}_{\mathrm{B}}^{2}=\left(\sigma_{\mathrm{g}}^{2} \mathrm{x} / \sigma_{\mathrm{g}}^{2} \mathrm{x}+\sigma_{\mathrm{e}}^{2} \mathrm{x}\right) \mathrm{x} 100
$$

where $\sigma_{\mathrm{g}}^{2} \mathrm{x}$ and $\sigma_{\mathrm{g}}^{2} \mathrm{y}$ are the genotypic variances of characters $\mathrm{x}$ and $\mathrm{y}$ respectively; $\sigma_{\mathrm{e}}^{2} \mathrm{x}$ and $\sigma_{\mathrm{e}}^{2} \mathrm{y}$ are the environmental variances for characters $\mathrm{x}$ and $\mathrm{y}$ while $\sigma_{\mathrm{g}} \mathrm{xy}$ and $\sigma_{\mathrm{e}} \mathrm{xy}$ are the genotypic and environmental covariance of character $\mathrm{x}$ and $\mathrm{y}$ respectively. A path analysis was used to determine the direct and indirect effects of some characters on pod yield following the procedure outlined by Dewey and Lu (1959).

\section{Results}

The mean square values for the studied characters and yield are shown in Table 3. Highly significant effect of locations and genotypes were recorded for all the studied characters and yield. Significant mean squares were also recorded for genotype $\mathrm{x}$ location for all the studied characters except, for height per plant at flowering that recorded highly significant mean square. Very large genotype mean squares were recorded for height per plant at flowering (4279.02), days to maturity (1106.50), pods per plant (9951.59), final height per plant (971.34) and sample seed weight (606.74).

The genotypic, phenotypic coefficient of variations (CV's) and broad sense heritability estimate are presented in Table 4. The characters exhibited a wide and continuous variation across varieties. All the studied characters exhibited different ranges in the two environments. Genotypic and phenotypic coefficients of variation were generally low except, for number of pods per plant, height per plant at flowering, final plant height, number of 
leaves per plant and yield per plant in the first environment. Low genotypic and phenotypic CV's were also recorded for all studied characters except number of pods per plant in the second environment. Genotypic CV was generally higher than phenotypic CV for all the studied characters in the two environments.

Table 3. Analysis of variance for quantitative characters and yield in Groundnut

\begin{tabular}{|c|c|c|c|c|c|c|c|c|c|c|c|c|}
\hline \multirow[b]{2}{*}{ Sources } & \multirow[b]{2}{*}{$\mathrm{DF}$} & \multicolumn{11}{|c|}{ Characters } \\
\hline & & $\begin{array}{c}\text { Days to } \\
50 \% \\
\text { flowering }\end{array}$ & $\begin{array}{l}\text { Height/ } \\
\text { plant at } \\
\text { flowering }\end{array}$ & $\begin{array}{c}\text { Number of } \\
\text { leaves/ } \\
\text { plant at } \\
\text { flowering }\end{array}$ & $\begin{array}{l}\text { Days to } \\
\text { maturity }\end{array}$ & $\begin{array}{c}\text { Final } \\
\text { height/ } \\
\text { plant }\end{array}$ & $\begin{array}{l}\text { Branches/ } \\
\text { plant at } \\
\text { maturity }\end{array}$ & $\begin{array}{l}\text { Nodes on } \\
\text { the main } \\
\text { stem/ } \\
\text { plant at } \\
\text { flowering }\end{array}$ & $\begin{array}{l}\text { Stem } \\
\text { girth/ } \\
\text { plant at } \\
\text { maturity }\end{array}$ & Pods/ plant & $\begin{array}{c}\text { Sample } \\
\text { seed } \\
\text { t weight } \\
(100 \\
\text { seeds) }\end{array}$ & $\begin{array}{l}\text { Yield/ } \\
\text { plant }\end{array}$ \\
\hline Replication & 2 & 0.02 & 0.43 & 0.45 & 0.04 & 3.21 & 0.18 & 1.99 & 0.02 & 24.15 & 0.55 & 1.24 \\
\hline Genotype (G) & 21 & $91.65 * *$ & $49.21 * *$ & $4279.02 * *$ & $1106.50 * *$ & $971.34 * *$ & $2.47 * *$ & $39.23 * *$ & $0.21 * *$ & $9951.59 * *$ & $666.74 * *$ & $219,51 * *$ \\
\hline Location (L) & 1 & $157.09^{* *}$ & $3346.16^{* *}$ & $654.82 * *$ & $447.34 * *$ & $536.43 * *$ & $38.19 * *$ & $411.63 * *$ & $18.49 * *$ & $8636.24 * *$ & $164.71 * *$ & $44.94 * *$ \\
\hline$G \times L$ & 21 & $0.66^{*}$ & $31.33 *$ & $388.20 * *$ & $7.48^{*}$ & $422.85^{*}$ & $2.35^{*}$ & $29.30 *$ & $0.13^{*}$ & $1636.77^{*}$ & $2.17^{*}$ & $79.06^{*}$ \\
\hline Error & 84 & 0.03 & 0.3 & 6.65 & 0.02 & 2.4 & 0.09 & 1.16 & 0.01 & 13.39 & 0.36 & 0.96 \\
\hline
\end{tabular}

$*, * *=$ Significant at $\mathrm{P} \leq 0.05$ and $\mathrm{P} \leq 0.01$ respectively

Table 4. Genotypic coefficient of variability ( $\mathrm{gCV}$ ), phenotypic coefficient of variability (pCV) and broad sense heritability $\left(\mathrm{h}^{2} \mathrm{~B}\right)$ for eleven groundnut traits in two environments

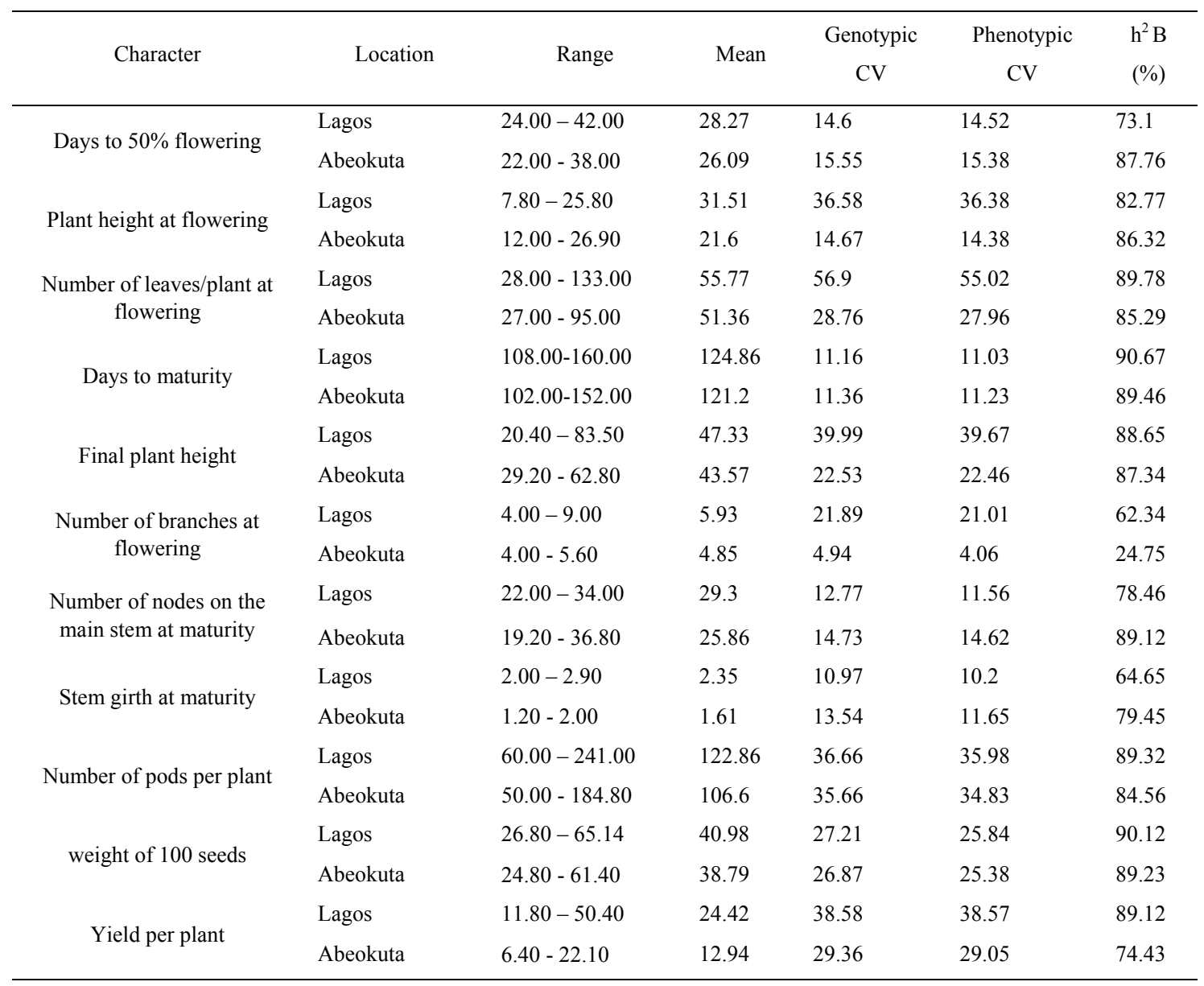


Table 5. Phenotypic correlation coefficients among eleven characters of groundnut genotypes in two locations

\begin{tabular}{|c|c|c|c|c|c|c|c|c|c|c|c|}
\hline Characters & Location & $\begin{array}{l}\text { Plant } \\
\text { height at } \\
\text { flowering }\end{array}$ & $\begin{array}{c}\text { Number of } \\
\text { leaves at } \\
\text { flowering }\end{array}$ & $\begin{array}{l}\text { Days to } \\
\text { maturity }\end{array}$ & $\begin{array}{c}\text { Final } \\
\text { plant } \\
\text { height }\end{array}$ & $\begin{array}{c}\text { Number of } \\
\text { branches at } \\
\text { flowering }\end{array}$ & $\begin{array}{c}\text { Nodes on } \\
\text { the main } \\
\text { stem at } \\
\text { maturity }\end{array}$ & $\begin{array}{c}\text { Stem } \\
\text { girth at } \\
\text { maturity }\end{array}$ & $\begin{array}{c}\text { Number } \\
\text { of pods/ } \\
\text { plant }\end{array}$ & $\begin{array}{c}\text { weight } \\
\text { of } 100 \\
\text { seeds }\end{array}$ & $\begin{array}{l}\text { Yield/ } \\
\text { plant }\end{array}$ \\
\hline $\begin{array}{c}\text { Days to } \\
50 \%\end{array}$ & 1 & -0.25 & $0.33 *$ & $0.68 * *$ & $0.31 *$ & -0.11 & -0.18 & 0.1 & -0.23 & $0.41 * *$ & 0.04 \\
\hline flowering & 2 & -0.01 & 0.18 & $0.64 * *$ & 0.28 & -0.02 & $-0.46 * *$ & 0.11 & -0.23 & $0.48 * *$ & 0.04 \\
\hline Plant height & 1 & & 0.04 & $0.30^{*}$ & $0.34 *$ & -0.19 & 0.09 & 0.01 & -0.06 & 0.12 & 0.26 \\
\hline at flowering & 2 & & 0.09 & 0.05 & 0.05 & -0.13 & 0.14 & 0.1 & -0.12 & $0.37^{*}$ & 0.13 \\
\hline $\begin{array}{c}\text { Number of } \\
\text { leaves at }\end{array}$ & 1 & & & 0.26 & $0.46^{* *}$ & 0.08 & $-0.36 * *$ & -0.15 & 0.19 & 0.08 & 0.13 \\
\hline flowering & 2 & & & 0.14 & 0.08 & 0.08 & -0.09 & -0.17 & 0.11 & $0.31^{*}$ & 0.16 \\
\hline Days to & 1 & & & & -0.05 & -0.11 & -0.24 & 0.15 & $-0.43 * *$ & $0.35^{* *}$ & $-0.48^{* *}$ \\
\hline maturity & 2 & & & & $-0.37^{*}$ & $-0.33 *$ & $-0.47 * *$ & 0.01 & $-0.42 *$ & $0.32 *$ & $-0.39 * *$ \\
\hline Final plant & 1 & & & & & $0.35^{* *}$ & $0.47 * *$ & 0.18 & 0.11 & $-0.37 * *$ & $0.29 *$ \\
\hline height & 2 & & & & & $0.38 * *$ & $0.66^{* *}$ & $0.40 * *$ & 0.23 & -0.19 & $0.34 * *$ \\
\hline $\begin{array}{c}\text { Number of } \\
\text { branches/ } \\
\text { plant at }\end{array}$ & 1 & & & & & & 0.21 & 0.25 & 0.09 & -0.12 & $0.31 *$ \\
\hline flowering & 2 & & & & & & $0.40^{* *}$ & 0.07 & 0.13 & -0.02 & 0.28 \\
\hline $\begin{array}{c}\text { Nodes on } \\
\text { the main } \\
\text { stem at }\end{array}$ & 1 & & & & & & & 0.24 & 0.18 & $0.35^{*}$ & 0.14 \\
\hline maturity & 2 & & & & & & & $0.55^{* *}$ & $0.38^{* *}$ & 0.24 & 0.25 \\
\hline Stem girth & 1 & & & & & & & & -0.09 & 0.04 & 0.22 \\
\hline at maturity & 2 & & & & & & & & $0.54 * *$ & 0.08 & $0.43^{* *}$ \\
\hline $\begin{array}{c}\text { Number of } \\
\text { pods per }\end{array}$ & 1 & & & & & & & & & -0.27 & $0.67 * *$ \\
\hline plant & 2 & & & & & & & & & -0.23 & $0.71 * *$ \\
\hline weight of & 1 & & & & & & & & & & 0.13 \\
\hline 100 seeds & 2 & & & & & & & & & & 0.05 \\
\hline
\end{tabular}

$*, * *=$ Significant at $\mathrm{P} \leq 0.05, \mathrm{P} \leq 0.01$ respective. $1=$ first location and $2=$ second location

Very high heritability estimates were recorded for majority of characters in both environments. Estimates ranged between $62.34 \%$ and $90.67 \%$ in the first environment, between $24.75 \%$ and $89.46 \%$ in the second environment for number of branches per plant at flowering and days to $50 \%$ flowering respectively. In the first environment, heritability estimate was moderately high for number of branches per plant at flowering (62.34\%), high for days to $50 \%$ flowering $(73.10 \%)$ and number of nodes on the main stem at maturity $(78.46)$ and very high estimates, above $80 \%$, for the remaining parameters. In the second environment, number of branches per plant at flowering recorded low heritability estimate $(24.75 \%)$, high estimates were observed for stem girth at maturity $(79.45 \%)$ and yield per plant $(74.43 \%$ ), while, very high heritability estimates (above $80 \%$ ) were recorded for the remaining studied characters.

The result of phenotypic correlation coefficient between characters in the two environments is presented in Table 5. Yield per plant had significant positive correlation with number of pods per plant $(0.67,0.71)$ and final height per plant $(0.29,0.34)$ in both environments. It showed positive significant correlation with number of branches per plant (0.31) in the first environment and stem girth per plant (0.43) in the second environment. The genotypic correlation coefficients between characters in the two environments are presented in Table 6. In most cases, characters exhibited similarity in both the direction and magnitude of correlation coefficients compared to 
phenotypic correlation coefficient. Generally, however, more parameters exhibited significant phenotypic correlation than the genotypic correlation. All characters exhibited non significant genotypic correlations with yield per plant except, number of pods per plant $(0.65,0.64)$. The environmental correlation coefficients between the characters for the two environments are presented in Table 7. Except in few cases characters exhibited similar environmental correlation in both environments though at different levels of significance. Number of pods per plant had highly significant positive environmental correlation with yield $(0.95 .0 .96)$ in the two environments, stem girth/ plant (0.76) in the second environment. It exhibited non-significant positive correlations with height per plant at flowering $(0.47,0.26)$, days to maturity $(0.17,0.03)$ and sample seed weight $(0.43$ and 0.12$)$ in the two environments.

Table 6. Genotypic correlation coefficients among eleven characters of groundnut genotypes in two locations

\begin{tabular}{|c|c|c|c|c|c|c|c|c|c|c|c|}
\hline Characters & Location & $\begin{array}{l}\text { Plant height } \\
\text { at flowering }\end{array}$ & $\begin{array}{c}\text { Number of } \\
\text { leaves at } \\
\text { flowering }\end{array}$ & $\begin{array}{l}\text { Days to } \\
\text { maturity }\end{array}$ & $\begin{array}{l}\text { Final } \\
\text { plant } \\
\text { height }\end{array}$ & $\begin{array}{c}\text { Number of } \\
\text { branches at } \\
\text { flowering }\end{array}$ & $\begin{array}{c}\text { Nodes on } \\
\text { the main } \\
\text { stem at } \\
\text { maturity }\end{array}$ & $\begin{array}{c}\text { Stem girth } \\
\text { at } \\
\text { maturity }\end{array}$ & $\begin{array}{c}\text { Number } \\
\text { of pods/ } \\
\text { plant }\end{array}$ & $\begin{array}{l}\text { weight } \\
\text { of } 100 \\
\text { seeds }\end{array}$ & $\begin{array}{l}\text { Yield/ } \\
\text { plant }\end{array}$ \\
\hline \multirow{2}{*}{$\begin{array}{c}\text { Days to } 50 \% \\
\text { flowering }\end{array}$} & 1 & -0.27 & 0.32 & $0.68 * *$ & -0.31 & -0.2 & -0.18 & -0.1 & -0.22 & $0.45^{*}$ & -0.05 \\
\hline & 2 & -5 & 0.14 & $0.64 * *$ & -0.3 & 0.15 & $-0.47 *$ & -0.04 & -0.26 & $0.47 *$ & -0.04 \\
\hline \multirow{2}{*}{$\begin{array}{l}\text { Plant height at } \\
\text { flowering }\end{array}$} & 1 & & 0.02 & 0.32 & -0.29 & -0.19 & -0.09 & 0.04 & -0.12 & 0.04 & 0.23 \\
\hline & 2 & & 0.13 & 0.12 & 0.04 & -0.11 & -0.04 & 0.1 & 0.08 & 0.37 & 0.08 \\
\hline \multirow{2}{*}{$\begin{array}{l}\text { Number of } \\
\text { leaves at } \\
\text { flowering }\end{array}$} & 1 & & & 0.25 & $-0.47^{*}$ & 0.04 & -0.28 & -0.15 & -0.2 & 0.1 & 0.14 \\
\hline & 2 & & & 0.06 & -0.05 & 0.22 & 0.03 & 0.15 & 0.17 & 0.28 & 0.24 \\
\hline \multirow{2}{*}{$\begin{array}{l}\text { Days to } \\
\text { maturity }\end{array}$} & 1 & & & & 0.04 & 0.1 & -0.05 & 0.3 & $-0.42 *$ & 0.39 & -0.33 \\
\hline & 2 & & & & -0.37 & -0.17 & $-0.47^{*}$ & 0.1 & $-0.48 *$ & 0.31 & -0.41 \\
\hline \multirow{2}{*}{$\begin{array}{c}\text { Final plant } \\
\text { height }\end{array}$} & 1 & & & & & $0.44 *$ & $0.62 * *$ & 21 & 0.15 & -0.36 & -0.26 \\
\hline & 2 & & & & & 0.04 & $0.71^{* *}$ & 0.32 & 0.24 & -0.21 & -0.36 \\
\hline \multirow{2}{*}{$\begin{array}{c}\text { Number of } \\
\text { branches/ plant } \\
\text { at flowering }\end{array}$} & 1 & & & & & & $0.44 *$ & -0.23 & 0.09 & -0.25 & 0.35 \\
\hline & 2 & & & & & & -0.05 & -0.04 & -0.01 & 0.33 & 0.17 \\
\hline \multirow{2}{*}{$\begin{array}{l}\text { Nodes on the } \\
\text { main stem at } \\
\text { maturity }\end{array}$} & 1 & & & & & & & $0.43^{*}$ & 0.11 & -0.38 & -0.34 \\
\hline & 2 & & & & & & & $0.57 * *$ & 0.42 & -0.26 & -0.25 \\
\hline \multirow{2}{*}{$\begin{array}{l}\text { Stem girth at } \\
\text { maturity }\end{array}$} & 1 & & & & & & & & 0.24 & 0.07 & 0.31 \\
\hline & 2 & & & & & & & & $0.45^{*}$ & 0.07 & 0.2 \\
\hline \multirow{2}{*}{$\begin{array}{c}\text { Number of } \\
\text { pods per plant }\end{array}$} & 1 & & & & & & & & & -0.28 & $065^{* *}$ \\
\hline & 2 & & & & & & & & & -0.24 & $0.64 * *$ \\
\hline \multirow{2}{*}{$\begin{array}{l}\text { weight of } 100 \\
\text { seeds }\end{array}$} & 1 & & & & & & & & & & 0.11 \\
\hline & 2 & & & & & & & & & & 0.12 \\
\hline
\end{tabular}

$*, * *=$ Significant at $\mathrm{P} \leq 0.05, \mathrm{P} \leq 0.01$ respectively. $1=$ first location and $2=$ second location. 
Table 7. Environmental correlation coefficients among eleven characters of groundnut genotypes grown in two locations

\begin{tabular}{|c|c|c|c|c|c|c|c|c|c|c|c|}
\hline Characters & Location & $\begin{array}{c}\text { Plant height } \\
\text { at } \\
\text { flowering }\end{array}$ & $\begin{array}{c}\text { Number of } \\
\text { leaves at } \\
\text { flowering }\end{array}$ & $\begin{array}{l}\text { Days to } \\
\text { maturity }\end{array}$ & $\begin{array}{l}\text { Final } \\
\text { plant } \\
\text { height }\end{array}$ & $\begin{array}{c}\text { Number of } \\
\text { branches at } \\
\text { flowering }\end{array}$ & $\begin{array}{c}\text { Nodes on } \\
\text { the main } \\
\text { stem at } \\
\text { maturity }\end{array}$ & $\begin{array}{c}\text { Stem girth } \\
\text { at } \\
\text { maturity }\end{array}$ & $\begin{array}{c}\text { Number } \\
\text { of pods/ } \\
\text { plant }\end{array}$ & $\begin{array}{l}\text { weight } \\
\text { of } 100 \\
\text { seeds }\end{array}$ & $\begin{array}{l}\text { Yield/ } \\
\text { plant }\end{array}$ \\
\hline \multirow{2}{*}{$\begin{array}{c}\text { Days to } 50 \% \\
\text { flowering }\end{array}$} & 1 & $0.79 * *$ & -0.09 & $0.89 * *$ & $0.94 * *$ & $0.62 *$ & 0.32 & -0.22 & -0.31 & -0.44 & -0.24 \\
\hline & 2 & 0.44 & $-0.90 * *$ & $0.99 * *$ & $0.64 *$ & $0.61 *$ & $0.55^{*}$ & -0.21 & -0.06 & 0.23 & -0.08 \\
\hline \multirow{2}{*}{$\begin{array}{l}\text { Plant height at } \\
\text { flowering }\end{array}$} & 1 & & $0.60^{*}$ & $0.58^{*}$ & $0.65 * *$ & 0.41 & 0.18 & 0.02 & $0.58^{*}$ & $0.64 * *$ & 0.47 \\
\hline & 2 & & 0.3 & $0.49^{*}$ & 0.08 & $0.60^{*}$ & 0.41 & 0.05 & 0.27 & $0.68^{* *}$ & 0.26 \\
\hline \multirow{2}{*}{$\begin{array}{l}\text { Number of } \\
\text { leaves at } \\
\text { flowering }\end{array}$} & 1 & & & -0.24 & -0.04 & $0.69 * *$ & 0.15 & -0.21 & $-0.74 * *$ & $0.61 *$ & $-0.70 * *$ \\
\hline & 2 & & & $-0.91 * *$ & $-0.59 *$ & $0.54^{*}$ & $0.54^{*}$ & -0.41 & -0.42 & 0.22 & -0.25 \\
\hline \multirow{2}{*}{$\begin{array}{l}\text { Days to } \\
\text { maturity }\end{array}$} & 1 & & & & $-0.84 * *$ & 0.08 & -0.02 & -0.07 & -0.03 & -0.09 & 0.17 \\
\hline & 2 & & & & $-0.64 *$ & $0.62 *$ & $-0.58^{*}$ & -0.25 & -0.1 & -0.17 & 0.03 \\
\hline \multirow{2}{*}{$\begin{array}{c}\text { Final plant } \\
\text { height }\end{array}$} & 1 & & & & & 0.07 & 0.47 & 0.21 & -0.12 & $0.52 *$ & -0.11 \\
\hline & 2 & & & & & 0.19 & $0.88^{* *}$ & $0.72 * *$ & -0.3 & $0.56^{*}$ & -0.26 \\
\hline $\begin{array}{c}\text { Number of } \\
\text { branches/ } \\
\text { plant at }\end{array}$ & 1 & & & & & & -0.28 & $-0.66 * *$ & -0.24 & -0.4 & -0.13 \\
\hline flowering & 2 & & & & & & -0.25 & -0.13 & -0.14 & -0.17 & -0.2 \\
\hline \multirow{2}{*}{$\begin{array}{l}\text { Nodes on the } \\
\text { main stem at } \\
\text { maturity }\end{array}$} & 1 & & & & & & & 0.44 & -0.3 & $-0.63 *$ & $-0.52 *$ \\
\hline & 2 & & & & & & & $0.82 * *$ & -0.37 & -0.11 & -0.36 \\
\hline \multirow{2}{*}{$\begin{array}{l}\text { Stem girth at } \\
\text { maturity }\end{array}$} & 1 & & & & & & & & 0.33 & 0.08 & 0.46 \\
\hline & 2 & & & & & & & & $0.77 * *$ & 0.25 & $0.76^{* *}$ \\
\hline \multirow{2}{*}{$\begin{array}{c}\text { Number of } \\
\text { pods per plant }\end{array}$} & 1 & & & & & & & & & 0.32 & $0.95^{* *}$ \\
\hline & 2 & & & & & & & & & 0.17 & $0.96 * *$ \\
\hline \multirow{2}{*}{$\begin{array}{l}\text { weight of } 100 \\
\text { seeds }\end{array}$} & 1 & & & & & & & & & & 0.43 \\
\hline & 2 & & & & & & & & & & 0.44 \\
\hline
\end{tabular}

*,** = Significant at $\mathrm{P} \leq 0.05, \mathrm{P} \leq 0.01$ respectively. $1=$ first location and $2=$ second location.

The direct and indirect effects of studied characters on yield per plant are presented in Table 8. In the first environment, number of pods per plant recorded the largest positive direct effect $(0.66)$ on yield per plant while, days to maturity had the largest $(0.68)$ indirect positive contribution to yield and a large indirect effect through reduction in number of leaves per plant at flowering $(-0.46)$. In the second environment number of pods per plant also exhibited the largest direct positive effect $(0.70)$ on yield per plant. Days to maturity also recorded the largest direct negative effect (-0.36) and largest positive indirect effect (0.68) on yield per plant. Days to maturity $(0.68,0.64)$ and sample seed weight $(0.42,0.48)$ had highly significant indirect effect on yield in the two environments while, number of nodes on the main stem at maturity showed highly significant indirect effect $(-0.47)$ on yield in the second environment with final height per plant contributing the largest indirect positive effect (0.68). 
Table 8. Direct and indirect effects of some characters on yield in twenty two genotypes of groundnut grown in two locations

\begin{tabular}{|c|c|c|c|c|c|c|c|c|c|c|c|c|c|}
\hline \multirow[b]{2}{*}{ Character } & \multirow[b]{2}{*}{ Location } & \multirow[b]{2}{*}{$\begin{array}{c}\text { Direct } \\
\text { effect } \\
\text { on } \\
\text { yield/ } \\
\text { plant }\end{array}$} & \multicolumn{11}{|c|}{ Indirect effect on yield/ plant through } \\
\hline & & & $\begin{array}{c}\text { Days to } \\
50 \% \\
\text { flowering }\end{array}$ & $\begin{array}{c}\text { Plant } \\
\text { height at } \\
\text { flowering }\end{array}$ & $\begin{array}{c}\text { Number } \\
\text { of leaves } \\
\text { at } \\
\text { flowering }\end{array}$ & $\begin{array}{l}\text { Days to } \\
\text { maturity }\end{array}$ & $\begin{array}{c}\text { Final } \\
\text { plant } \\
\text { height }\end{array}$ & $\begin{array}{c}\text { Number } \\
\text { of } \\
\text { branches } \\
\text { at } \\
\text { flowering }\end{array}$ & $\begin{array}{c}\text { Nodes } \\
\text { on the } \\
\text { main } \\
\text { stem at } \\
\text { maturity }\end{array}$ & $\begin{array}{c}\text { Stem } \\
\text { girth at } \\
\text { maturity }\end{array}$ & $\begin{array}{c}\text { Number } \\
\text { of pods/ } \\
\text { plant }\end{array}$ & $\begin{array}{l}\text { weight } \\
\text { of } 100 \\
\text { seeds }\end{array}$ & $\begin{array}{l}\text { Genotypic } \\
\text { correlation }\end{array}$ \\
\hline $\begin{array}{c}\text { Days to } \\
50 \%\end{array}$ & 1 & 0.04 & - & $-0.25^{*}$ & $0.33 * *$ & $0.68 * *$ & $-0.31 *$ & -0.14 & -0.2 & 0.1 & -0.23 & $0.42 * *$ & -0.05 \\
\hline flowering & 2 & 0.04 & - & $-0.30^{*}$ & 0.17 & $0.64 * *$ & $-0.29^{*}$ & 0.03 & $-0.47 * *$ & 0.09 & -0.24 & $0.48^{* *}$ & -0.04 \\
\hline Plant & 1 & $0.25^{*}$ & -0.25 & - & 0.03 & 0.11 & $-0.32 *$ & -0.19 & -0.03 & 0.02 & -0.08 & 0.09 & 0.23 \\
\hline $\begin{array}{l}\text { height at } \\
\text { flowering }\end{array}$ & 2 & 0.12 & -0.3 & - & 0.11 & 0.08 & $-0.45^{* *}$ & -0.13 & -0.03 & 0.11 & -0.11 & $0.37 * *$ & 0.08 \\
\hline $\begin{array}{l}\text { Number } \\
\text { of leaves }\end{array}$ & 1 & -0.14 & $0.33 * *$ & 0.03 & - & $0.26^{*}$ & $-0.46^{* *}$ & 0.06 & $-0.33 * *$ & 0.15 & -0.19 & 0.09 & 0.14 \\
\hline $\begin{array}{c}\text { at } \\
\text { flowering }\end{array}$ & 2 & -0.19 & 0.17 & 0.11 & - & 0.12 & -0.07 & 0.01 & -0.06 & 0.17 & -0.13 & $0.31 *$ & 0.24 \\
\hline Days to & 1 & $-0.33 * *$ & $0.68^{* *}$ & 0.11 & $0.26^{*}$ & - & -0.05 & 0.11 & -0.17 & 0.2 & $-0.39 * *$ & $0.37 * *$ & -0.33 \\
\hline maturity & 2 & $-0.36^{* *}$ & $0.64 * *$ & 0.08 & 0.12 & - & $-0.37 * *$ & $0.28 *$ & $-0.47^{* *}$ & 0.03 & $-0.44 * *$ & $0.32 *$ & -0.41 \\
\hline $\begin{array}{l}\text { Final } \\
\text { plant }\end{array}$ & 1 & $-0.25^{*}$ & $-0.31 *$ & $-0.32 *$ & $-0.46^{* *}$ & -0.05 & - & $0.38 * *$ & $0.52 * *$ & 0.19 & 0.12 & $-0.37 * *$ & -0.26 \\
\hline height & 2 & $-0.34 * *$ & $-0.29 *$ & $-0.45^{* *}$ & -0.07 & $-0.37 * *$ & - & $0.29 *$ & $0.68 * *$ & $0.37 * *$ & 0.24 & -0.2 & -0.36 \\
\hline $\begin{array}{c}\text { Number } \\
\text { of }\end{array}$ & 1 & $0.33 * *$ & -0.14 & -0.19 & 0.06 & 0.11 & $0.38 * *$ & - & $0.30 *$ & 0.24 & 0.09 & -0.16 & 0.35 \\
\hline $\begin{array}{l}\text { branches } \\
\text { at } \\
\text { flowering }\end{array}$ & 2 & $0.24 *$ & 0.03 & -0.13 & 0.01 & $0.28^{*}$ & $0.29 *$ & - & $0.27^{*}$ & 0.04 & 0.1 & -0.11 & 0.17 \\
\hline $\begin{array}{l}\text { Nodes on } \\
\text { the main } \\
\text { stem at }\end{array}$ & 1 & -0.21 & -0.2 & -0.03 & $-0.33^{* *}$ & -0.17 & $0.52 * *$ & $0.30^{*}$ & - & 0.2 & 0.15 & $-0.36^{* *}$ & -0.34 \\
\hline maturity & 2 & $-0.25^{*}$ & $-0.47 * *$ & -0.03 & -0.06 & $-0.47 * *$ & $0.68 * *$ & $0.27 *$ & - & $0.55^{* *}$ & $0.39^{* *}$ & $-0.25^{*}$ & -0.25 \\
\hline Stem & 1 & $-0.25^{*}$ & 0.1 & 0.02 & 0.15 & 0.2 & 0.19 & 0.24 & 0.2 & - & 0.14 & 0.02 & 0.31 \\
\hline $\begin{array}{l}\text { girsth at } \\
\text { maturity }\end{array}$ & 2 & $-0.35^{* *}$ & 0.09 & 0.11 & 0.17 & 0.03 & $0.37 * *$ & 0.04 & $0.55^{* *}$ & - & $0.51 * *$ & 0.08 & 0.2 \\
\hline Number & 1 & $0.66^{* *}$ & -0.23 & -0.08 & -0.11 & $-0.39 * *$ & 0.12 & 0.09 & 0.15 & 0.14 & - & $-0.28 *$ & $0.65 * *$ \\
\hline $\begin{array}{c}\text { of pods/ } \\
\text { plant }\end{array}$ & 2 & $0.70 * *$ & -0.24 & -0.11 & -0.19 & $-0.44 * *$ & 0.24 & 0.1 & $0.39 * *$ & $0.51 * *$ & - & -0.23 & $0.64 * *$ \\
\hline $\begin{array}{c}\text { Weight of } \\
100\end{array}$ & 1 & 0.12 & $0.42 * *$ & 0.09 & 0.09 & $0.37 * *$ & $-0.37 * *$ & -0.16 & $-0.36^{* *}$ & 0.02 & $-0.28 *$ & - & 0.11 \\
\hline Seeds & 2 & 0.07 & $0.48 * *$ & $0.37 * *$ & $0.31 *$ & $0.32 *$ & -0.2 & -0.11 & $-0.25^{*}$ & 0.08 & -0.23 & - & 0.12 \\
\hline
\end{tabular}

$*, * *=$ Significant at $\mathrm{P} \leq 0.05, \mathrm{P} \leq .01$ respectively. $1=$ first location and $2=$ second location.

\section{Discussion}

The existence of genetic variation can be employed as the basis for improving yield and other potentials of crop plant (Morakinyo \& Makinde, 1991; Muhammad et al., 2007; Jonah et al., 2010). Results of this investigation revealed that the groundnut genotypes investigated exhibited wide range of genetic variations in most agronomic parameters (Table 3). These observed wide ranges and significant variation between characters measured on the groundnut genotypes are indicative of the wealth of the studied population as a source of genes for future improvement programme. The higher genotypic variation relative to the phenotypic counterparts implies less environmental influence on the characters under study (Table 4). The similarity in genotypic and phenotypic variation of most characters in the two environments is consistent with the autogamous nature of groundnut. In addition, the genotypes must have attained homozygosity at various loci and would be expected to be fairly similar across environments. However, the pronounced differences observed in yield, final plant height and a 
number of other attributes over the environments is an indication that the characters are under both additive and multiplicative gene and environmental effects. Similar observation had been reported earlier (Mishra \& Chhonkar, 1977; Kato \& Takeda, 1996; Rao et al., 1997).

Correlations are a measure of the intensity of association between variables (Lukhele, 1981; Jogloy et al., 2011). In selection for yield, the mode of inheritance of yield components must be understood; correlation among them and between each component and yield will provide information on the mode of inheritance of yield. Correlation by contrast indicates whether two variables are interdependent or vary together hence, it is a measure of closeness of association. Similar findings have been reported by Ntare and Williams (1998) in groundnut and by Ariyo (1987b), Makinde (1988), Board et al. (1999) and Ojo (2003) in other crops. It is an established fact that phenotypic character expression incorporates both genotypic and environmental effects. Therefore, the non-significant genotypic correlation between any two characters, relative to its significant phenotypic counterpart, is indicative of appreciable environmental effect. Generally, genotypic relationships are of premium importance in plant breeding. The significant genotypic correlation between yield and number of pods per plant in both environments indicates its importance in any groundnut improvement or breeding programme (Table 6). This observation corroborates that of Jogloy et al. (2011) that there is possibility to simultaneously improve number of pods per plant, seed size and pod yield in groundnut and Haro et al. (2007) that seed number is generally associated with seed yield rather than weight of individual seeds. The significant genotypic correlation between number of nodes on the main stem at maturity and final plant height implied that number of nodes on the main stem could be increased by selecting for taller genotypes (Table 6). However, negative correlation of yield with days to $50 \%$ flowering, days to maturity, final plant height and number of nodes on the main stem indicates that breeding programme aimed at improving yield must use groundnut genotypes that are early maturing and dwarf. The latter corroborates that of et al. (1978) and Burow et al. (2004) that days to first harvest correlated with pod yield negatively in okra and groundnut respectively. Positive genotypic correlation between yield per plant and stem girth at maturity, number of leaves per plant, weight of 100 seeds and number of branches per plant at flowering implied that they constituted the components of yield in this population of groundnut. Consequently, direct selection for these characters in the early segregating generations will be effective and can be exploited for genetic improvement of yield among wide collection of groundnut germplasm (Ojo, 2003).

The observed negative direct effects of days to maturity, nodes on the main stem at maturity and stem girth at maturity suggested that selection on the basis of these traits might lead to loss in terms of seed yield (Table 8). This observation corroborates that of Muhammad et al. (2007); Arshad et al. (2006). Days to $50 \%$ flowering, plant height at flowering, number of branches at flowering, number of pods per plant and sample seed weight had positive direct effect on yield in both locations, suggested important selection criteria in groundnut. Similar observation has been reported by Jogloy et al. (2011), Das et al. (1989) and Arslan (2005).

\section{Conclusion}

The observed high level of variation within the groundnut genotypes used, coupled with high broad sense heritability suggested that genetic improvement and selection procedure in this population of groundnut will be worthwhile. The characters evaluated in this study were quite variable and exhibited higher genotypic correlation relative to the phenotypic correlation indicating less environmental influence. Although some characters varied in performance in the two environments, most characters exhibited similar environmental correlation indicating that their performance would be fairly consistent from one environment to another.

\section{Acknowledgement}

The authors acknowledged with thanks the management of the Lagos State University, Nigeria for providing enabling environment to work as research scientists. Contributions of Miss. Akinsuyi Tope and Olanrewaju Bukola during manuscript preparation are highly appreciated. While, that of Mr. Alex Akhalume in data analysis is posthumously acknowledged.

\section{References}

Ariyo, O. J. (1987a). Multivariate analysis and the choice of parents for hybridization in okra (Abelmoschus esculentus (L.) Moench). Theor. Appl. Genet., 74, 361-363. http://dx.doi.org/10.1007/BF00274718

Ariyo, O. J. (1987b). Stability of performance of okra as influenced by planting date. Theor. Appl. Genet., 74, 83-86. http://dx.doi.org/10.1007/BF00290088

Arshad, M., Naazar, A., \& Abdul, G. (2006). Character correlation and path coefficient in soybean (Glycine max (L.) Merill). Pak. J. Botany, 38(1), 121-130. 
Arslan, M., (2005). Effects of haulm cutting time on haulm and pod yield of peanut. J. Agron., 4(1), 39-43. http://dx.doi.org/10.3923/ja.2005.39.43

Board, J. E., Kang, M. S., \& Harville, B. G. (1999). Path analysis of the yield formation processes for late-planted soybean. Agro. J, 19, 128-135. http://dx.doi.org/10.2134/agronj1999.00021962009100010020x

Burrow, M. D., Lopez, Y., Baring, M. R., Ayers, J. L., \& Simpson, C. E. (2004). Breeding for early-maturing peanut. Proceedings of the American Peanut Research and Education Society, Inc., July 8-11, 2003, Clearwater Beach, Florida. pp. 26-27.

Clay, H. S., \& Dombek, D. (1995). Comparing soyabean cultivar ranking and selection for yield with AMMI and full data performance estimates. Crop Science. 35, 1536-1541. http://dx.doi.org/10.2135/cropsci1995.0011183X003500060003x

Culp, T. W., Bailey, W. K., \& Hanmons, R. O. (1968). Natural Hybridization of peanuts. Crop Science, 8, 109-111. http://dx.doi.org/10.2135/cropsci1968.0011183X000800010033x

Das, M. L., Rahman, A., \& Miah, A. J. (1989). Correlation, path-coefficient and regression studies in soybean. Bangladesh J. Agric. Research, 14(1), 27-29.

Dewey, D. R., \& Lu, K. H. (1959). A correlation and path- coefficient analysis of components of crested wheat $\begin{array}{lllll}\text { grass } & \text { seed } & \text { pgron. } & J & \end{array}$ http://dx.doi.org/10.2134/agronj1959.00021962005100090002x

Falconer, D. S. (1989). Introduction to quantitative genetics. (3rd ed.). Essex: Longman Scientific and Technical, p.438.

Gibbons, J. A., \& Tatterfield, T. (1969). Outcrossing trial with groundnut, (Arachis hypogaea L.). Rhodesia Journal of Agricultural Research, 7, 71-85.

Gomez, K. A., \& Gomez, A. A. (1984). Statistical procedures for Agricultural Research. (2nd ed.). Canada: John Willey \& Sons Inc. p.680.

Haro, R. J., Otegui, M. E., Collino, D. J., \& Dardenelli, J. L. (2007). Seed yield determination and radiation use efficiency in irrigated peanut crops: Response to temperature and source-sink ratio variations. Field Crops Res., 103, 217-228. http://dx.doi.org/10.1016/j.fcr.2007.06.004

Jain, S. K., \& Workman, P. L. (1966). The genetic of inbreeding species. Advanced genetics, 14, 55-131.

Jogloy, C., Jaisil, P., Akkasaeng, C., Kesmala, T., \& Jogloy, S. (2011). Heritabilty and correlation for maturity and pod yeild in peanut. Journal of Applied Sciences Research, 7(2), 134-140.

Jonah, P. M., Adeniji, O. T., \& Wammanda, D. T. (2010). Variability and genetic correlations for yield and yield characters in some Bambara groundnut (Vigna subterranea) cultivars. Int. J. Agric. Biol., 12, 303-307.

Kato, T., \&Takeda, K. (1996). Associations among characters related to yield sink capacity in space-planted rice. Crop Science, 36, 1135-1139. http://dx.doi.org/10.2135/cropsci1996.0011183X003600050011x

Kaul, T., Lal, G., \& Peter, K. V. (1978). Correlation and path-coefficient analysis of component of earliness pod yield and yield in okra. Indian J. Agric. Sci, 48(8), 459-463.

Kowal, J. M., \& Kassan, A. H. (1978). Agricultural ecology of savannah: A study of West Africa. (p. 318). Oxford: Clarendon Press.

Lukhele, P. E. (1981). Estimation of genetic variability in sorghum (Sorghum bicolor (L.) Moenel). Unpublished M. Sc. Thesis, Faculty of Agriculture, Ahmadu Bello University. Nigeria.

Makinde, S. C. (1988). Genetic characterization of okra (Abelmoschus esculentus (L.) Moench.) cultivars. Unpublished M. Sc. Thesis, Faculty of Science, University of Ilorin, Ilorin. Nigeria.

Mishra, R. S., \& Chhonkar, V. S. (1977). Genetic divergence in okra. Indian J. Agric. Sci., 49, 247-249.

Morakinyo, J. A., \& Makinde, S. C. (1991). Variability and heritability in local cultivars of okra (Abelmoschus esculentus (L.) Moench.). Nig. J. of Botany, 4, 33-40.

Muhammad, F. A. M., Muhammad, A., Afsari, S. Q., \& Abdul, G. (2007). Assessment of genetic variability, correlation and path analyses for yield and its componentsn in soybean. Pak. J. Bot., 39(2), 405-413.

Ntare, B. M., \& Williams, J. H. (1998). Heritability and genotype x environment interaction for yield and component of yield in populations of groundnut under semi-arid condition. African Crop Science Journal, 6(2), 119-127. http://dx.doi.org/10.4314/acsj.v6i2.27807 
Ojo, D. K. (2003). Environmental variability, correlation, genetic determination and contribution of nine agronomic traits to seed yield in selected tropical soybean (Glycine max (L) Merr.) genotypes. ASSET series A, 3(4), 127-136.

Purseglove, J. W. (1975). Dicotyledons: in Tropical Crops. (pp. 199-232). London: Longman.

Rao, S. A., Khan, M. A., McNeilly, T., \& Khan, A. A. (1997). Cause and effect relations of yield and yield components in rice (Oriza sativa L.). J. Genet. and Breed, 51, 1-5.

Singh, M. (1991). Genotypic and phenotypic correlations in plant traits. Syria International Centre for Agricultural Research. In the Day Areas. p. 277.

Smith, B. W. (1950). Arachis hypogaea; Arid flower and substerranean fruit. American Journal of Botany, 27, 803- 815.

Swaminatham, M. S., Austin, A., Kaul, A. K., \& Naik, M. S. (1969). New approaches to breeding for improved plant protein Vienna: IAEA. p.132

White, J. W., \& Gonzalez, A. (1990). Characterization of negative association between seed yield and seed size among genotypes of common bean. Field crops Research, 23, 159-175. http://dx.doi.org/10.1016/0378-4290(90)90052-D

Wright, F., \& Dobzhansky, T. H. (1970). Genetics of evolution process. (p. 234). New York: Columbia University Press. 